\title{
Le laboratoire du LCIE à Fontenay-aux-Roses
}

\author{
G. LE ROY
}

\section{Introduction}

Les évolutions ont été très rapides et les changements les plus importants dans l'activité de dosimétrie du LCIE ont concerné le passage de l'âge de l'imprimerie (les fameuses plaques zinc) et du tout manuel à la gestion informatisée. Le remplacement du film par une autre technique n' aurait pas pu avoir lieu avant cette informatisation.

Aujourd'hui, le service de dosimétrie du LCIE n'existe plus en tant que tel : son activité a été intégrée en 2002 dans la société « LCIE Landauer » devenue en 2010 «Landauer Europe ».

\subsection{Début de la dosimétrie au LCIE}

La création de cette activité n'était pas récente puisqu'elle datait de 1941, et ceci à la demande de la Chambre syndicale des constructeurs d'appareils d'électroradiologie. Cette section spécialisée avait pour objet la vérification de la conformité aux normes des appareils générateurs de rayons $\mathrm{X}$ ainsi que des appareils d'électricité médicale : appareils de radiothermie à haute fréquence, générateurs d'ultrasons, électrocardiographes, etc.

Pourquoi les industriels de la radiologie ont-ils demandé cela à un laboratoire d'électricité comme le LCIE ? La réponse est simple : ils se sont adressés à un laboratoire d'électricité car en 1941 la mesure des courants faibles était difficile. C'était le cas du courant d'ionisation produit dans une chambre d'ionisation et de sa traduction en roentgens.

La formule de la loi d' Ohm « $V=R I »$ en considérant « $R I »$ pour rayonnements ionisants peut expliquer aussi ce choix. Pour exemple le volume de collection pouvait atteindre $2000 \mathrm{~cm}^{3}$ et dans ces conditions le courant d'ionisation était de l'ordre de $2 \times 10^{-8} \mathrm{~A}$, facilement mesurable au moyen d'un galvanomètre à aiguille dont le spot lumineux était projeté sur un mur afin d'augmenter sa sensibilité. 
Par la suite, vers 1957, et du fait de l'importance croissante des besoins de protection contre les radiations, les pouvoirs publics ont organisé au sein de l'Institut national d'hygiène (INH) un « Service central de protection contre les rayonnements ionisants » et le texte du décret de création indiquait une liaison avec les organismes existants. Cette façon de faire a donc été suivie par le LCIE et le Professeur Louis Bugnard, Directeur de l'Institut national d'hygiène (INH). Une convention a été signée entre l'INH et le LCIE ; l'INH portant ses efforts sur l'étude et la réalisation de nouveaux étalons, le LCIE se consacrant plus spécialement aux opérations courantes, d'une part, de vérification des appareils et moyens de mesure et, d'autre part, d'analyse et dosage des radiations. Ainsi André Allisy de l'INH a travaillé sur la mesure des doses et conçu une nouvelle chambre absolue basée sur les travaux de Lauriston Taylor du Laboratoire primaire américain National Bureau of Standards (NBS).

Les deux figures suivantes (10.1 et 10.2) montrent une partie des installations du LCIE en 1957.

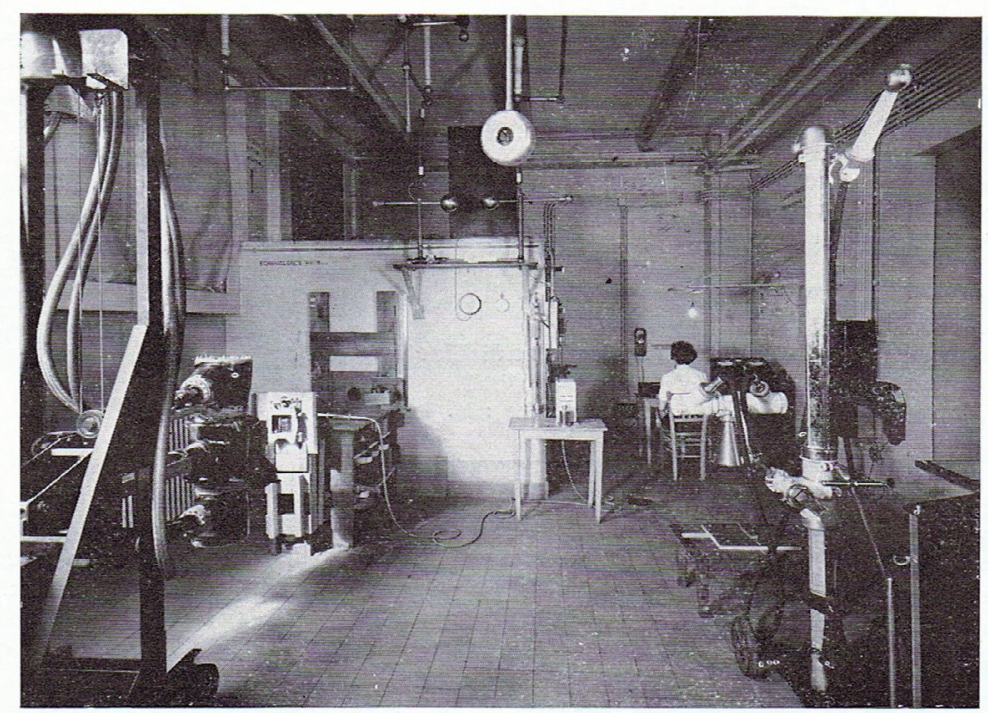

Figure 10.1 - Salle de dosimétrie et de radiométallographie (SFE, 1957).

À gauche, générateur $250 \mathrm{kV}$ en courant continu ; au centre, grande chambre d'ionisation ; à droite, générateur $120 \mathrm{kV}$ en courant alternatif ou redressé. 


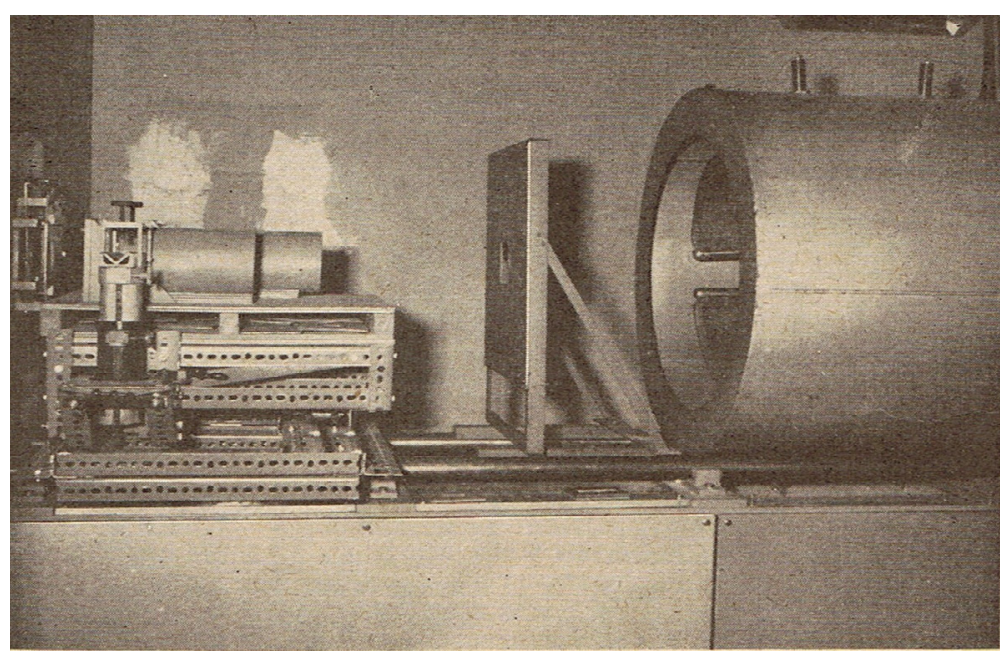

Figure 10.2 - Vue de la grande chambre d'ionisation et du support de fixation des dosimètres (LCIE, 1958a).

Le mesureur de courant associé utilise le principe de la balance de Townsend et donc permet de mesurer des courants faibles générés dans un petit volume de collection de $5 \mathrm{~cm}^{3}$.

La chambre d'ionisation à plaques parallèles (free air chamber), dite aussi « chambre Allisy », a été fabriquée en deux modèles par la société Baudoin : une pour le LCIE et une autre pour l'ETCA (Établissement central des armées) situé à Arcueil.

En parallèle et à la même époque, nul n'ignorait les effets physiologiques des rayonnements et l'intérêt d'en connaître les doses. Aussi le Commissariat à l'énergie atomique avait développé et mis au point des films photographiques adaptés à la détection des rayonnements (voir chapitre 8).

À cette époque, le Ministère de la santé publique a publié pour la France (Journal officiel du 11 juillet 1957) une « circulaire relative aux recommandations générales visant la protection contre les radiations ionisantes », dans laquelle il était indiqué que «pour les travailleurs professionnels exposés, la dose maximale admissible hebdomadaire peut atteindre 0,3 rem pendant quelques semaines à condition que la dose cumulée pendant une année n'excède pas 5 rem ».

Aussi, en accord avec l'Institut national d'hygiène et avec l'aide du CEA, le LCIE a donc créé en 1958 un service chargé de fournir aux particuliers, aux 
industriels et aux administrations les films nécessaires et d'en assurer le développement et l'interprétation physique. Ce service « Dosimétrie des films » a été mis place par Étienne Pierre, en liaison technique avec le CEA. Les dosimètres photographiques mis à disposition des clients répondaient à ces exigences.

La figure 10.3 montre un dosimètre sous pochette soudée avec un film Kodak type 3 inséré dans un porte-écrans étain-cadmium.

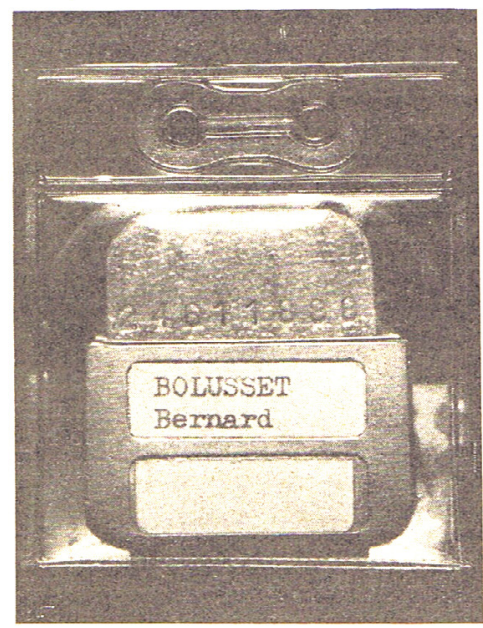

Figure 10.3 - Vue d'un film de détection (méthode utilisée par le CEA) (voir chapitre 8). Le film est engagé aux deux tiers dans son porte-écran étain-cadmium.

Sur la partie nue, on remarque le numéro d'identification. L'ensemble est contenu dans une pochette soudée de chlorure de vinyle.

Lorsque le nouveau film Kodak type 1 (figure 10.4) conçu par Norbert Chassende-Baroz et le CEN/FAR a été mis sur le marché, le LCIE a remplacé le film Kodak type 3 (deux films de sensibilité différente dans la même pochette). Ainsi le nombre de films à développer a été divisé par deux et la lecture du marquage moins aléatoire (voir chapitre 2).

Le porte-écrans « cadmium-étain » a été remplacé par un porte-écrans « cuivreplomb » plus léger mais toujours correspondant à un modèle CEA sur lequel le LCIE ajoutait une bande de plomb qui était collée par les opératrices du Service de dosimétrie des films. 


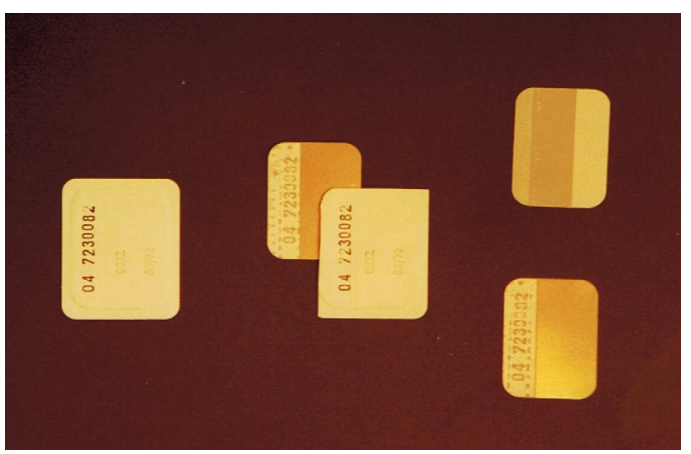

Figure 10.4 - Dosimètre Kodak T1 Chassende-Baroz avec sa numérotation.

Le tableau I ci-dessous présente les différents types de dosimétrie photographiques utilisés au LCIE entre 1958 et 1990.

TABLEAU I

Les dosimètres photographiques utilisés au LCIE.

\begin{tabular}{lll}
\hline Années approximatives & 1958 à 1960 & 1961 à 1990 \\
\hline film Kodak & Type 3 (2 films) & Type 1 en enveloppe étanche avec bande marquage \\
Porte-écrans & Etain/cadmium & Cuivre/plomb \\
Densitomètre & Analyse par transmission & Analyse par réflexion \\
\hline
\end{tabular}

Le film est inséré dans un porte-écrans définissant quatre plages de lecture sur le film développé.

1 - plage « nue »

2 - plage sous $0,2 \mathrm{~mm}$ de cuivre

3 - plage sous $0,6 \mathrm{~mm}$ de cuivre

4 - plage sous $0,2 \mathrm{~mm}$ de cuivre et $0,5 \mathrm{~mm}$ de plomb

Le porte-écrans est réversible; c'est-à-dire que des filtres de même nature sont placés en vis-à-vis et donc situés de part et d'autre du film.

Les masses surfaciques des écrans traversés par le rayonnement et délimitant chacune des plages de lecture sont indiquées dans le tableau II. La plage «nue » a une épaisseur de matière placée devant les émulsions réduite à la pochette en plastique, l'étiquette et l'enveloppe du film Kodak. Cette épaisseur est aussi prise en compte dans le calcul des masses surfaciques des écrans traversés par le rayonnement. 
Tableau II

Masses surfaciques des écrans.

\begin{tabular}{lccc}
\hline Filtres avant & Filtres arrière \\
\hline Matériel des plages & $\begin{array}{c}\text { Masse surfacique } \\
\left(\mathrm{mg} / \mathrm{cm}^{2}\right)\end{array}$ & Matériel des plages & $\begin{array}{c}\text { Masse surfacique } \\
\left(\mathrm{mg} / \mathrm{cm}^{2}\right)\end{array}$ \\
«nue » & 75 & «nue » & 75 \\
$0,2 \mathrm{~mm}$ de cuivre & 250 & $0,2 \mathrm{~mm}$ de cuivre & 250 \\
$0,6 \mathrm{~mm}$ de cuivre & 610 & $0,6 \mathrm{~mm}$ de cuivre & 610 \\
$0,5 \mathrm{~mm}$ de plomb & 800 & $0,5 \mathrm{~mm}$ de plomb & 800 \\
$+0,2 \mathrm{~mm}$ de cuivre & & $+0,2 \mathrm{~mm}$ de cuivre & \\
plage dite « sous plomb» & & plage dite « sous plomb » \\
\hline
\end{tabular}

Il est à noter que le porte-écrans est constitué d'un support en aluminium de 0,6 mm d'épaisseur maintenant les différents filtres à leur place respective. La partie supérieure du porte-écrans, correspondant à la superposition de ce support de $0,6 \mathrm{~mm}$ d'aluminium et du filtre de $0,2 \mathrm{~mm}$ de cuivre, est utilisée comme une zone de détection pour les rayonnements $\mathrm{X}$ de très basse énergie.

L'équivalent de dose est déterminé à partir des valeurs des densités optiques mesurées sur le film et ceci sur les plages délimitées par le porte-écrans sur ce dernier.

La mesure pour les rayonnements gamma se fait sur la plage dite « sous plomb » de $800 \mathrm{mg} / \mathrm{cm}^{2}$ qui répond aux conditions d'équilibre électronique pour les faisceaux de cobalt 60 et de césium 137.

\subsection{Ma participation à la dosimétrie au LCIE}

En 1968, je travaillais dans le service du CEN/FAR/STEP (Service technique d'études et de protection) dirigé par Henri Francois. Le sujet de mon étude portait sur le comptage par un système électrique de détecteurs de traces alpha. Puis en 1969, j'ai été embauché par Serge Leuzinger pour intégrer la Section dosimétrie qui était en contact avec Henri Francois. Mon activité portait sur les étalonnages de dosimètres associés aux installations de radiothérapie à l'aide des générateurs à rayons $\mathrm{X}$ de $200 \mathrm{kV}$ ou la mise en place de nouveaux moyens de mesure pour la radiologie.

L'activité d'étalonnage concernait plus de 200 dosimètres intégrateurs par an et ils avaient pour noms : Dosix (fabrication par CGR), Electra Hammer 5 R, Massiot 2 R, Microdosimètre Massiot, Philips puis Baldwin, Farmer, PTW 
Universel. Les dosimètres intégrateurs, qui avaient été réglés dans le faisceau de rayons X du LCIE, étaient ensuite connectés aux générateurs de radiothérapie ; le médecin affichait un nombre de «top » correspondant au nombre de « roentgen » à délivrer et le dosimètre coupait l'émission de rayons $\mathrm{X}$ lorsque la dose était atteinte.

À cette époque, le LCIE fabriquait, comme le CEN/FAR (Raymond Prigent), des mesureurs de courant en utilisant des lampes électromètres, des bases de temps... En fait tout ce qui n'existait pas sur le marché.

Le mesureur de courant de l'installation des rayons X $50 \mathrm{kV}$ était un appareil construit par Massiot selon le principe de la balance de Townsend avec l'obligation d'avoir un opérateur qui compensait la charge de façon à garder le galvanomètre à zéro. Dix années après, un dosimètre Farmer, très prisé des radiophysiciens, fonctionna selon ce principe.

Ensuite, les électromètres utilisant des condensateurs vibrants ou des transistors à effet de champ ayant des gains très élevés sont apparus dans le commerce. Ce fut une vraie révolution et cela permit de mesurer des courants de $10^{-12} \mathrm{~A}$ avec un bruit de fond de l'électromètre de $10^{-15} \mathrm{~A}$.

Les résultats obtenus avec des chambres cavité type Bragg-Gray avaient conduit à mettre des anneaux de garde polarisés pour obtenir une valeur de courant identique dans les deux polarités. En fait, cela venait des électromètres qui n'étaient pas assez performants (gain trop faible).

J'ai donc travaillé aux améliorations des chambres à parois d'air ou à la création de nouvelles chambres d'ionisation destinées aux mesures de dose.

J'ai également participé à des études destinées au contrôle des installations de radiologie. En effet j'ai contribué à la mise en œuvre de méthodes de mesure (pour exemple: contrôle des hautes tensions à l'aide d'un radiochromomètre «cuivre/graphite» inspiré de celui de Benoist) pour la campagne lancée par le SCPRI et confiée au LCIE en 1969. Il fallait vérifier la sécurité et l'état des installations de radiologie médicales en service en France en sélectionnant un cabinet par type d'équipement. Les fabricants étaient nombreux à cette époque : CGR, Chenaille, Dutertre, Trophy, Tubix, Philips, Siemens, etc.

Cela m'a permis de faire des essais avec des générateurs ayant des kénotrons comme système de redressement, des barres HT avant leur remplacement par des câbles souples et d'utiliser une bonnette de Dessane pour la visualisation des fuites de gaines radiogènes. Dans ce domaine aussi l'évolution a été aussi très rapide (suppression des ouvertures de palpation des paravents, de la scopie, radiographie pulsée, cabine auto-protégée...). 
Les sites Internet consacrés à la radiologie et à ses débuts sont riches en enseignement (ci-après voiture rayons X de 1914 de Georges Massiot, figure 10.5). Il faut lire l'historique rédigé par Henri Nahun présent sur le site de la Société française de radiologie (SFR) : http://www.sfrnet.org.

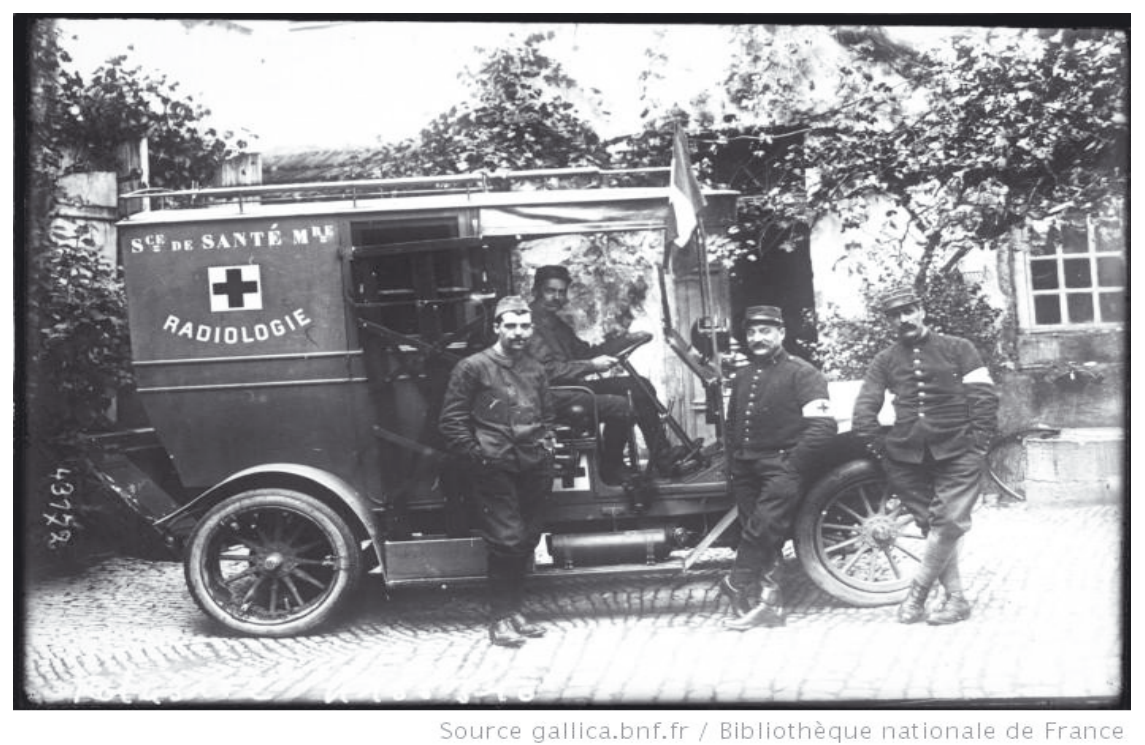

Figure 10.5 - Véhicule radiologique militaire de Georges Massiot.

Après ces détours, revenons à la dosimétrie photographique.

À mon arrivée au LCIE, ma participation à l'activité pour la dosimétrie photographique se limitait aux étalonnages des films étalons ou à des études particulières sur les porte-écrans et aussi aux réponses aux questions techniques posées par leur client : les lettres mettant transmises par Étienne Pierre. Il ne m'a demandé de prendre en charge l'activité de dosimétrie films que beaucoup plus tard. Cela a continué en 1984 par l'établissement d'un dossier d'une analyse fonctionnelle de l'activité en vue du passage à une informatisation des moyens d'impression. En même temps, les équipements techniques ont commencé à être améliorés (chambre noire avec commande numérique, soudeuse haute fréquence...). 


\subsection{Gestion du service de dosimétrie du LCIE de 1957 à 2001}

À mon arrivée au LCIE en 1969, le service de dosimétrie photographique du LCIE créé en 1957 avait toujours Étienne Pierre comme Chef de service et il était secondé par Georges Gallicier, responsable de la production.

«En 1957 le service comporte deux sections principales : la section «films vierges » qui prépare et distribue les films et la section « films portés » qui reçoit, développe et lit les films, et indique les doses reçues. De plus, un secrétariat est chargé de la diffusion des résultats. » Cette phrase est extraite d'un article paru dans un bulletin d'information du LCIE $\mathrm{N}^{\circ} 22$ de décembre 1958 (article de Étienne Pierre) (LCIE, 1958b).

Au début, le nombre de films traité était faible (200 à 500 par mois ou quinzaine). À mon arrivée au LCIE, la petite activité était devenue grande et traitait de l'ordre de 20000 dosimètres par mois. Le type de film avait changé (Toujours Kodak mais type 1 au lieu du type 3) ainsi que le porte-écrans. Ainsi les dosimètres étaient constitués d'un film Kodak type 1 inséré dans un porte-écrans « cuivre/plomb» : conditionnés sous pochettes scellées pour la plupart des abonnés mais aussi fournis pour une faible part en « films nus » $(10 \%$ mais uniquement pour les dosimètres de poitrine non associés à une émulsion nucléaire).

En 1969, l'organisation était maintenant constituée en équipes : une équipe « listage » pour l'impression des documents et la gestion des abonnements, une équipe «expédition-développement», une équipe «lecture », un secrétariat « courrier/résultats aux médecins du Travail » et une comptable travaillant chez elle à mi-temps. La salle d'impression était remplie de meubles à tiroirs contenant les plaques « zinc/porteur» gravées chacune avec le nom et le prénom d'un porteur ou l'intitulé « témoin ». Chaque entité était rangée par région, par type de dosimètre (poitrine, poignet) et chaque plaque « zinc » était classée dans l'ordre alphabétique (nom puis prénom) avec celle du « témoin » à la fin. Une machine munie d'un grand volant (une grosse pince Dymo) était utilisée pour emboutir les plaques « zinc » et constituer les plaques «zinc » pour chaque nouveau porteur. D'autres rangements contenaient les plaques «zinc/adresses d'envoi » servant à imprimer les en-têtes de bordereaux ainsi que les étiquettes destinés aux envois. Il en était de même pour les adresses de facturation. Il y avait deux jeux de porte-écrans par porteur muni de son étiquette avec son nom : celui porté et celui destiné à la période suivante. Les jeux de porte-écrans en attente étaient rangés sur des plateaux par type de dosimètre, par client, et dans l'ordre alphabétique des porteurs de ce client. L'équipe « listage » avait la charge d'enregistrer les nouveaux clients, les nouveaux porteurs, en leur attribuant un numéro d'identifiant (le fameux numéro LCIE) en l'écrivant sur un gros cahier et en établissant une fiche individuelle avec les numéros de 
sécurité sociale et destinée à être rangé dans les nombreux tiroirs et en suivant l'ordre alphabétique des noms et des prénoms. Avant d'affecter un numéro LCIE à un porteur, il fallait s'assurer que ce porteur n'avait pas déjà été inscrit au LCIE : dans ce cas l'ancien numéro était repris. En 1969, il y avait déjà plus de 100000 fiches de porteurs.

Les bordereaux étaient fournis par un imprimeur et étaient composés d'une liasse de cinq feuillets reprenant les indications générales sur chaque feuillet. Le premier feuillet devait être imprimé de façon à retrouver le texte sur les feuillets suivants. Ce travail se faisait avec les machines de listage qui recevaient alors des chargeurs plaque « zinc ». La numérotation des films de type 1 se faisait sur une machine rotative ayant un numéroteur à neuf chiffres avançant de un à chaque passage d'un film de type 1. Comme ce n'est pas une machine faite pour les films, il y avait des réglages difficiles et de nombreux films à mettre au rebut. La trame du numéro du film a été conçue pour le numéroteur (deux pour la période, un pour le type de dosimètre, un pour l'année, et enfin cinq pour la séquence de ce type de dosimètre soit les neuf chiffres du numéroteur).

Les modifications sont alors arrêtées pour la période à venir et cela entraînait la gravure des plaques des nouveaux inscrits, le retrait des plaques des annulations, la préparation ou le retrait des porte-écrans en attente, l'impression des bordereaux par type de dosimètres, l'impression des étiquettes d'envoi, etc. Un vrai travail de fourmis qui nécessitait « ordre, méthode, rigueur» dans tous les classements ou rangements. Je me rappelle qu'il y avait un jeu de cartes avec des noms et des prénoms qui servaient à tester les futures opératrices : la difficulté était de ne pas oublier de prendre en compte le prénom après le nom dans le classement alphabétique de ces fiches cartons. Les codes clients étaient propres au LCIE et avaient une racine $(7 \mathrm{C}, 7 \mathrm{CH}, 78 \mathrm{CH}, 9 \ldots)$ suivi d'un numéro d'ordre. Par exemple, $7 \mathrm{C}$ : dosimètres de type « 7 » sous pochette soudée destinés à un client de la région parisienne $7 \mathrm{CH}$ : dosimètres de type « 7 » sous pochette soudée destinés à un client hors région parisienne. Les bordereaux des codes « 7C » étaient imprimés client par client. Un premier passage avait lieu dans la machine de listage avec les plaques des adresses des clients. Puis un second passage dans la machine de listage chargée avec les plaques des porteurs, le numéro du film imprimé avançant avant chaque passage d'une plaque « zinc » et d'un bordereau.

Le premier feuillet du bordereau était ensuite détaché et transmis à l'équipe « expédition/développement » qui prenait les plateaux de porte-écrans ayant les noms des porteurs, insérait les films préalablement numérotés, soudait l'ensemble dans les pochettes et constituait les envois pour chaque client. Les dosimètres reçus après leur période de port étaient sortis de leur pochette, les porte-écrans rangés par clients, les films aussi, et traités en chambre noire. Les films "noirs » étaient analysés en premier pour faire rapidement les alertes « fortes doses ». Ensuite les 
films mis dans des supports étaient lus à l'œil par les opératrices « lecture » en se basant sur un support de films étalons et ceci pour les faibles valeurs de dose (inférieures à $100 \mathrm{mrem}$ ). Les doses étaient portées à la main sur le bordereau de retour. Tous les films et les résultats étaient contrôlés par le responsable du service. Ensuite une personne tapait à la machine les doses manuscrites sur le second feuillet du bordereau de ce client et l'adressait au médecin du Travail. En 1969, la saisie des doses destinées au SCPRI se faisait manuellement sur une perforatrice à bandes en code binaire selon un format conforme à l'attente de cet organisme. La personne responsable de la comptabilité préparait la facturation en copiant à la main sur une fiche client individuelle le nombre de films fournis chaque période de port à ce client et les comptaient en se référant au bordereau. Les fiches étaient marquées d'annotation en rouge ou vert selon les ajouts (frais particuliers) ou retraits à intégrer dans la facture faite par trimestre ou par semestre.

Vers 1970, la section de Dosimétrie photographie a été transférée du premier étage au second étage du LCIE en lui aménageant des espaces adaptés à son augmentation d'activité et aux machines d'impression et de soudage HF, la chambre noire restant toujours au premier étage dans son état ancien.

Au début des années 1980, le LCIE a reçu une lettre du SCPRI signée par le Professeur Pellerin à laquelle était jointe une grille de classification des clients du LCIE dans des catégories bien définies ( $\mathrm{P}$ pour les hôpitaux, $\mathrm{D}$ pour les dentistes, etc.), des définitions de fichiers pour les mouvements de porteurs (NXAB, ANAB, CHGNM, DOSES...). Les formats des fichiers étaient fixés par le SCPRI et ainsi que la date de la mise en place de son application sur des disquettes informatiques de sept pouces.

Le service de films a alors acheté une console de saisie ITT intégrant un écran de visualisation, un clavier et dans laquelle il fallait insérer une disquette de sept pouces pour ensuite faire la saisie manuellement. Pour ce faire, la nouvelle classification demandée par le SCPRI (M, D, N, P, Q, X, Z suivi d'un numéro dans chaque séquence) avait été affectée à chaque numéro d'abonnement $(7 \mathrm{CP}, 7 \mathrm{CH}$, etc.) imprimée sur les bordereaux et inscrits sur les fiches cartonnées des porteurs. Le service de films fonctionnait alors sans aucun équipement informatique exception faite de la console ITT utilisée comme une machine à écrire. Elle permettait de visualiser toutes les pistes des disquettes sept pouces. C'était un appareil peu courant. Il m'a permis de rencontrer Jacques Ballay du service informatique de SCPRI car je lui avais donné accès à cet ITT afin de visualiser les pistes de ses propres disquettes non accessibles avec l'équipement du Vésinet. Puis le SCPRI demanda au LCIE de ne lui transmettre que les doses des dosimètres de poitrine supérieures à 30 mrem et ceci sans doute par manque de place sur leur ordinateur. Le fichier « Doses » fut donc réduit en taille. 
Le service de dosimétrie avait des contacts avec les autres laboratoires de dosimétrie, donnant lieu à des visites respectives de nos installations et aussi à l'échange des bonnes adresses. Cela m'a permis de tisser des liens amicaux avec ces laboratoires ayant les mêmes préoccupations techniques ou de fournitures (films, machines...). Ainsi au CEN/FAR/STEP, j'ai eu accès à leurs équipements automatisés qui étaient toujours à la pointe de la technique et ceci par des échanges avec Gui Portal, Francis Bermann, Léon Faye, Yvon Magri et plus tard avec Francis Leblanc de l'IPSN. En parlant de cette équipe, je pense aussi aux échanges amicaux et techniques avec Jean-Louis Chartier et Christian Itie, tous deux travaillant sur des installations de rayons $\mathrm{X}$ du CEN/FAR implantées dans les sous-sols du LCIE. Pour l'ETCA, mes visites à Arcueil m'ont permis de voir un beau laboratoire de dosimétrie et de rencontrer Martine Guilland et Josette Briand-Champlong ainsi que le fils de Norbert Chassende-Baroz. J'ai aussi rencontré plusieurs fois les responsables des Établissements COGEMA de La Hague (René Prevot puis Hubert Truffert) et de Marcoule (Michel Espagnan) au cours d'opérations d'étalonnage ou des fameuses réunions du GTN1 ayant pour sujet les nouvelles grandeurs opérationnelles et leurs concepts (NGO) et pour animateur Jean-Louis Chartier. J'ai aussi travaillé en liaison avec le laboratoire d'EDF de Saint-Denis et vu leur chambre noire équipée d'un robot de développement ainsi que leurs automates mis en place par la société ACMA. Je me souviens de leur machine rotative de marquage par jet d'encre des pochettes des films Kodak type 2 associée à une générateur de rayons X marquant le film par un codage binaire. De même, j'ai eu des échanges fructueux avec le service de films du CNRS de Saclay François Clapier puis Isabelle Vabre. En qui concerne le SCPRI, je peux dire que je n' ai pas eu la chance de visiter le laboratoire du Vésinet. J'ai rencontré les Professeurs Pierre Pellerin, Jean Chanteur et Jean-Pierre Moroni et lors de réunions BNM ou de commissions du FRETAC (organisme appelé maintenant Comité français d'accréditation COFRAC). Les échanges avec le SCPRI ont toujours eu lieu par téléphone avec Alain Biau. Ma première rencontre a eu lieu en 2000 lors de la réception d'un équipement faite par l'OPRI chez un constructeur installé en province : Alain Biau était accompagné de Marc Valero. Cet industriel présentait, avant leur livraison, les nouveaux robots de traitement des films commandés par le laboratoire du Vésinet et avait invité, en autres, le LCIE, avec l'idée de vendre des équipements similaires. Enfin le LCIE effectuait des étalonnages de «Dosifilms ${ }^{\circledR}$ » pour le laboratoire Massiot d'Ailly et de ce fait, je connaissais par téléphone les responsables d'Ailly-le-Haut Clocher (80), Philippe Pellissier, ainsi que son support technique, Dominique Jaegle.

Ce rappel de ces contacts montre que la dosimétrie des films était un milieu où tout le monde se connaissait et s'échangeait des adresses de fournisseurs. Il était souvent fait appel à la société Cauvi spécialisée dans la fourniture de matériels de 
laboratoires photographiques pour les besoins en chambre noire (CEN/FAR, ETCA, EDF, LCIE...). Ainsi la société de robotique ACMA (une filiale de Renault) avait invité le LCIE avec d'autres laboratoires pour montrer des robots dans leur siège de Flins et ensuite en fonctionnement chez EDF à Saint-Denis.

Il faut savoir que le service de dosimétrie photographie créé en 1957 a vu son nombre de clients augmenter petit à petit et que la façon de travailler est restée la même car une automatisation complète était difficile sans changer les porte-écrans LCIE par un modèle à géométrie identique de façon être manipuler par des robots. Le remplacement complet d'un double jeu de porte-écrans entraînait une dépense très importante. Pour mémoire, à Saint-Denis, le modèle initial de porte-écrans des dosimètres EDF été remplacé afin de rendre possible l'automatisation du conditionnement.

Le travail était très manuel et de ce fait nécessitait d'avoir recours à du personnel intérimaire durant les périodes d'été et d'absence de personnel. Il en était de même pour le laboratoire du CEN/FAR/STEP et leur besoin de remplacement passait par le LCIE qui lui détachait du personnel pour le traitement des films. De même, le CEN/STEP possédait une salle équipée de microscopes, située dans les locaux du LCIE, pour la lecture de ses émulsions nucléaires NTA. Le service de dosimétrie du LCIE avait accès à cette salle pour compter ses propres films NTA.

Tout cela pour montrer que le Service de dosimétrie films du LCIE était très en retard en ce qui concerne les automatisations et l'informatisation. Les films étaient gérés manuellement comme une grande collection de timbres et ceci jusqu'à l'archivage des films lus. Une fois l'installation terminée au second étage du laboratoire, les efforts ont porté d'une part, sur la partie technique liée aux porteécrans et au développement des films et d'autre part, sur la migration vers une gestion informatisée.

\section{Au sujet des porte-écrans}

Afin d'améliorer la détection des rayonnements bêta, la possibilité d'équiper le porte-écrans « cuivre/plomb » d'une plage pour ces rayonnements a été étudiée. Des étalonnages ont alors été effectués avant ajoutant une plage « aluminium » et ceci dans des faisceaux de rayonnements bêta: prométhéum 147, thallium 204 et strontium 90.

Ces essais ont montré que le support en aluminium de 0,6 mm d'épaisseur maintenant les différents filtres à leur place respective faisait office de plage d'aluminium sur le film développé Kodak type 1. Cela a été ensuite vérifié lors du passage au film Kodak type 2. Par la suite, les dosimètres exposés en rayonnement 
bêta ou avec des électrons (accident de Forbach, voir chapitre 7) ont continué à être détectés avec cette zone ; la mesure de la densité optique étant faite sur la plage « nue » et reportée sur une courbe des densités optiques mesurées sous la plage dite «sous plomb» lors des étalonnages en rayonnement gamma du cobalt 60. Cette estimation n'a de sens qu'en présence d'une irradiation en «bêta pur » car la quantification de la part bêta est souvent difficile à évaluer si le film a été aussi exposé en rayons $\mathrm{X}$.

Des dosimètres de type 7 ont permis d'annoncer aussitôt après leur développement une forte irradiation en bêta lors de l'accident de Forbach. J'ai téléphoné au SCPRI et au Directeur de la société (en absence du médecin du travail) pour leur dire qu'il y avait une très forte dose en bêta sur des films de porteurs. Le responsable de la société m'a répondu que cela n'était pas possible car il n'avait que des électrons... Bref, le film avait vu juste et Alain Biau a pris l'affaire en main (voir chapitre 7).

Le service avait besoin de répondre à certains clients qui voulaient avoir des dosimètres détectant aussi les neutrons thermiques, c'est-à-dire avec une plage de cadmium qui, sous l'effet des neutrons thermiques, générait un rayonnement gamma marquant ainsi le film sous cette plage. Il a été envisagé deux possibilités : revenir au premier porte-écrans « étain-cadmium » pour ces clients ou contacter Gui Portal pour avoir l'autorisation d'utiliser un porte-écrans PS1 pour cette demande particulière. Je profite de ce roman « historique » pour le remercier à nouveau. Le LCIE a donc acheté au CEN/FAR un lot de porte-écrans PS1 qui était refusé sur leurs bancs robotisés par suite d'un défaut de forme non visible à l'œil et parfaitement utilisable pour un conditionnement fait à la main par le LCIE.

Puis vinrent les dosimètres type 1 (poitrine) et type 2 (poignet) mis au catalogue du LCIE. La demande principale venait de la société Schlumberger. Son personnel manipulant un gammadensimètre contenant une source américium-béryllium bénéficiait d'une surveillance dosimétrique pour les rayonnements gamma, neutrons thermiques et neutrons rapides. Il y avait donc dans la même pochette un dosimètre type 1 et un dosimètre type 5 (films NTA).

\section{Le développement des films}

Le développement des films se faisait dans une chambre noire (figure 10.6). Les films étaient passés dans la coupeuse par l'opératrice, mis dans des paniers de façon à constituer des cannes.

Les cannes étaient stockées dans un coffre aussi étanche à la lumière. Lorsque le nombre de cannes étaient suffisant, une opératrice les prenait une à une et les 


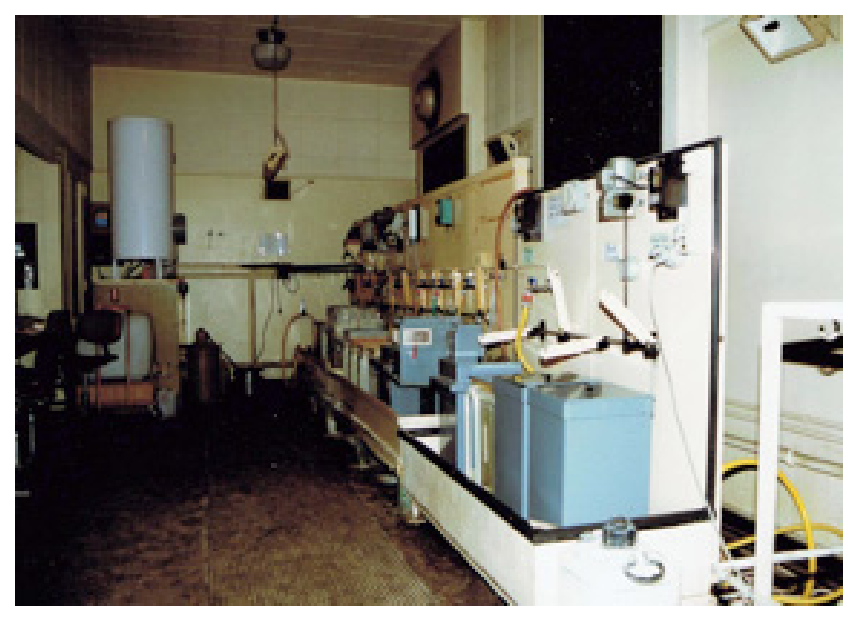

Figure 10.6 - Dispositif de développement en chambre noire.

passait manuellement d'un bac à l'autre : bac du révélateur, bac bain d'arrêt, bac fixateur et enfin les bacs de lavage. Le passage dans les sept bacs se faisait à la main, seule l'agitation dans les bacs se faisait automatiquement car chaque canne était accrochée à un bras agité par un moteur. Les cannes constituées de sept paniers pesaient sept $\mathrm{kg}$ chacun. Un développement de 12 cannes revenait à manipuler plus de $500 \mathrm{~kg}$.

La nouvelle chambre noire a été installée sous les combles alors que l'ancienne était située au premier étage et donc dans un espace à loyer élevé. Cela a permis de concevoir un ensemble très complet comprenant la chambre claire, le passage en chicane sans porte et enfin la partie la chambre noire avec un robot de développement. Après avoir vu différents bancs de développement il a été retenu la solution d'installer un robot fabriqué par la société ATN à commande numérique programmable assurant automatiquement les cannes d'une cuve à la suivante (figure 10.7). L'opératrice devait mettre régulièrement une canne sur la base de départ et retirer celle déposée sur la base d'arrivée. Tout l'ensemble mécanique (cuves, support etc.), exception faite du portique ATN et son pupitre de commande avait été fabriqué par l'atelier de mécanique du LCIE à partir de nos plans et avec une forte participation du chef d'atelier, Michel Hudry. La réalisation d'outillages spécifiques nécessaires à la dosimétrie ou l'entretien mécanique de certaines machines (coupeuses, soudeuses...) étaient confiés à cet atelier. Sa compétence était connue et de ce fait il assurait de nombreuses réalisations pour les activités LCIE-BNM ainsi que pour les besoins de Jean-Louis Chartier du CEN/FAR. 
Quatre nouvelles coupeuses ont été aussi achetées à la société Ravaj, aussi un fournisseur du CEN/FAR. René Rossignol, alors responsable de l'équipe « expédition/développement », avait une formation en mécanique industrielle et de ce fait a participé fortement à l'amélioration de ces coupeuses et à la mise en place du banc ATN.

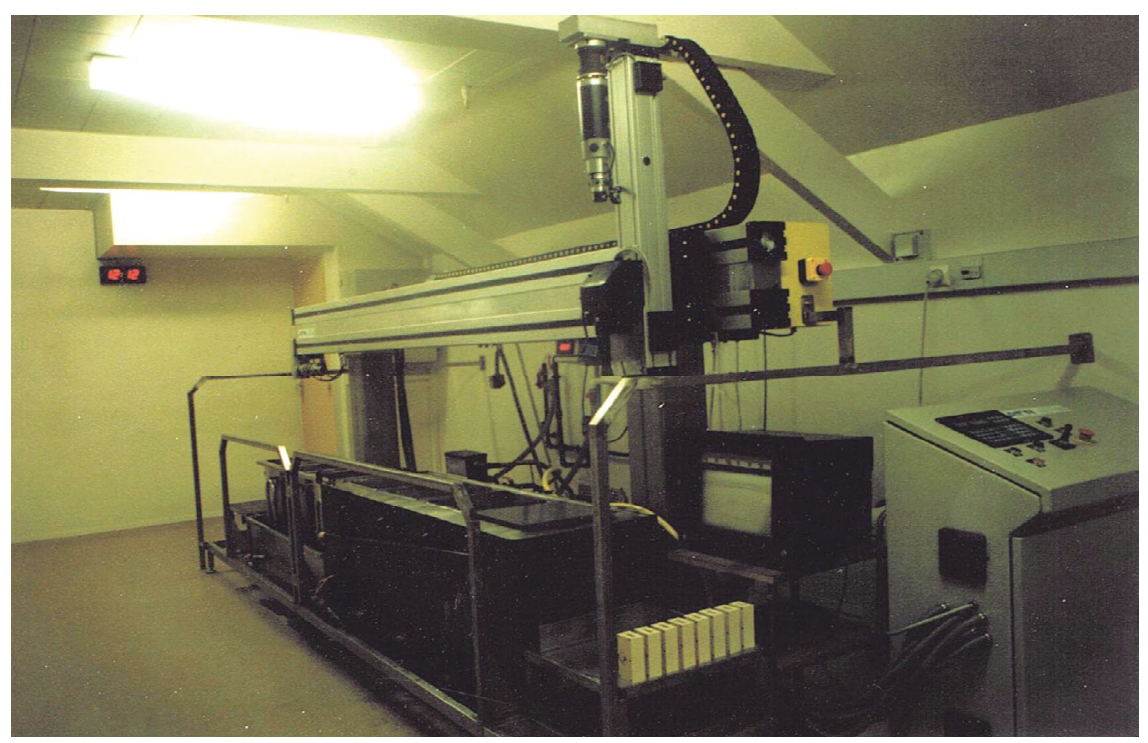

Figure 10.7 - Robot de développement des dosimètres.

Cette photo du robot (figure 10.7) a été prise au moment de sa mise en place avant l'installation des barrières de sécurité. Il est à noter que le banc de développement ne comporte pas de tunnel et que sa face avant permet un accès facile aux cuves. Cela a été conçu de façon à pouvoir assurer un transfert manuel des cannes d'une cuve à l'autre en cas de panne du robot. Cet ensemble a fonctionné jusqu'à la suppression des films.

\section{L'informatisation de la gestion}

En 1985, la gestion et les impressions des documents se faisaient toujours à l'aide de machine à plaques «zinc ». Les machines de listage étaient obsolètes et leur maintenance devenait difficile. Pour les visiteurs, l'impression des bordereaux rappelait les débuts de l'imprimerie et à la presse de Gutenberg ! Il faut savoir que la section « films » ne faisait pas partie des activités « nobles » du LCIE et que les 
investissements allaient plus vers les secteurs de l'électronique. Cette section a été maintenue au LCIE car elle avait des résultats financiers très importants servant à investir dans des domaines plus scientifiques et faisant appel à des ingénieurs et des techniciens supérieurs et non à du personnel sans formation de base. Aussi l'informatisation en partie de la section «films » a été lancée en 1984 en commençant par son analyse fonctionnelle et en établissant un cahier des charges prenant en compte les améliorations et les gains de production. Étienne Pierre et moi-même, nous sommes donc investis dans cette étude en impliquant fortement tout le personnel de la section. Il a été convenu d'informatiser la partie concernant la gestion sans modifier les analyses de films. Pour ce faire il a été acheté un ordinateur d'occasion IBM 34 avec une imprimante et 3 consoles. Le système a été programmé en GAP par Bernard Goin de la société COGITO. Un logiciel classique de comptabilité a été mis en service début 1985. Le transfert de tous les registres papier ou les fiches des porteurs a nécessité l'intervention d'opératrices de saisie. Les anciens codes clients ont été abandonnés afin de permettre la gestion des abonnements clients à l'aide de leur code SCPRI. De nombreux tests ont permis de montrer la cohérence entre les deux éditions de bordereaux pour la même période de port : manuellement à l'aide des plaques « zinc » et avec l'IBM 34.

Tous les dosimètres mensuels de la période de novembre 1985 ont été expédiés avant la fin du mois d'octobre via l'IBM 34 et le logiciel « DOSE». Pour des raisons pratiques liées à leur faible pourcentage, la gestion informatisée des abonnements des dosimètres portés deux mois avait été décalée début 1986. Cette mutation a été faite sur moins d'une année car elle ne concernait que les points suivants :

a. gestion des fichiers, des clients, des porteurs, impression des bordereaux d'accompagnement des dosimètres, impression des étiquettes destinées aux films ;

b. saisie des doses sur une console de l'IBM 34 à partir des valeurs inscrites à la main par les opératrices de l'équipe «lecture» sur les bordereaux d'accompagnement retournés avec les dosimètres ;

c. impression des rapports de contrôle, impression des factures ;

d. création de la disquette 7 pouces destinée au SCPRI.

La prévision des gains de temps s'était de suite confirmée du fait de la suppression de toutes les manipulations liées aux plaques «zinc». Aussi pour devancer ce changement, des intérimaires assuraient les opérations de conditionnement à certains postes de travail. Le marquage des films Kodak type 1 se faisait toujours sur la marqueuse rotative à neuf chiffres. Les bordereaux d'accompagnement une fois imprimés et les étiquettes des dosimètres à expédier étaient transmis aux opératrices de l'équipe «expédition » qui collaient chaque étiquette sur le film type 1 ayant le même numéro en l'insérant en même temps dans 
un porte-écrans : ceux-ci étant maintenant banalisés. Les dosimètres étaient ensuite soudés dans une pochette étanche avant leur expédition. Sur la figure 10.8 cidessous sont présentés tous les dosimètres mis à la disposition des clients, exception faite de ceux munis d'un porte-écrans PS1.

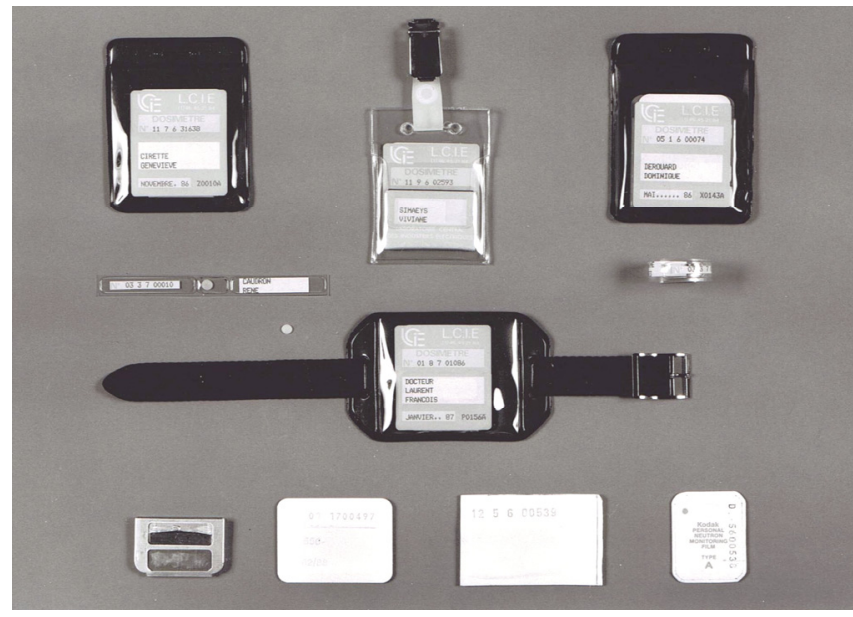

Figure 10.8 - Dosimètres de poitrine type 7 (en «films nus» type 9), les bagues de TLD type 3 et les émulsions nucléaires NTA type 5.

Pour mémoire, les bagues sont des pastilles de FLi 7 qui ont été achetées auprès de Philippe Blanchard qui a longtemps travaillé dans l'équipe de Gui Portal du CEN/FAR. Les lectures étaient faites avec un appareil TLD de marque Saphymo SRT 20 conçu à partir des études du CEN/FAR/STEP.

En outre, cette informatisation a permis de mettre en œuvre un jeu de six couleurs d'étiquettes de façon à faciliter les échanges des films chez les utilisateurs et aussi à leur retour. En effet, tous les films retournés étaient développés et analysés avec la transmission d'un rapport de contrôle pour tous ceux retournés dans les trois mois suivant la période de port. Les autres appelés «super-retard» étaient aussi développés et analysés mais seule une lettre d'information était transmis au médecin du travail pour ceux dépassant $1 \mathrm{mSv}$.

La gestion des abonnements a été assurée grâce à l'ordinateur IBM 34 pendant de nombreuses années mais son ancienneté posait parfois des problèmes de maintenance à IBM. Le changement d'ordinateur a été la conséquence de l'abandon de la fabrication du film Kodak type 1 qui avait déjà tendance à voir son prix augmenter depuis quelques années. Puis, Kodak ayant besoin de nouvelles 
machines dans son usine de Châlons-sur-Saône, son commercial est venu au LCIE pour proposer une très forte augmentation de tarif afin de pouvoir continuer la fabrication du fameux film type 1. En le raccompagnant je le lui ai indiqué notre refus et ceci avant d'en parler à la Direction du LCIE. Étienne Pierre avait pris sa retraite et maintenant la section « films » n'était plus rattachée aux transformateurs HT mais elle dépendait d'un chef de service responsable de la sécurité intrinsèque. Nos échanges étaient très courtois mais la section « films » restait une activité à part, en fait externe aux métiers du LCIE. De ce fait, $\mathrm{j}$ ' ai aussi des échanges directs avec le Directeur adjoint Edmond Beau. J'en profite pour le remercier pour le temps qu'il a consacré à cette section et à son appui pour trouver les circuits courts afin de répondre rapidement aux besoins d'investissement d'une activité très rentable. Il faut savoir que la section «films » devait avoir des résultats très positifs, sa comptabilité analytique incluant les investissements de matériels et de location des locaux. Son activité était en concurrence à l'époque avec le SCPRI, qui gardait les tarifs de ses dosimètres inchangés et qui, de plus, était exonéré de TVA par son statut. Notre autre concurrent était la société privée Philips (anciennement : Massiot puis Massiot-Philips). Son service «Dosifim» avait les mêmes impératifs de gestion que la section « films » du LCIE. L'autre point commun est qu'il faisait maintenant partie d'un groupe international dont le métier principal n'était pas la dosimétrie photographique.

Abandonner le type 1 pour un autre type de films était alors notre objectif. Les trois laboratoires utilisant le film type 1 (CEN/FAR, ETCA, CNRS) ont alors travaillé ensemble pour trouver la meilleure solution. Je me rappelle de la réunion organisée par Gui Portal. Le choix devait se faire entre les films Kodak type 2 (deux émulsions de part et d'autre d'un support transparent), les films Agfa (deux films dans la même pochette) et les films Fuji (trois films dans le même étui). Gui Portal a réussi à trouver comment obtenir des films Agfa en France grâce à ses contacts avec les laboratoires allemands.

Nous avons chacun étudié le meilleur choix pour nos laboratoires respectifs. Le CEN/FAR et l'ETCA ont choisi le film Agfa car ils avaient participé à l'équipement initial pour fabriquer le film type 1 avec Norbert Chassende-Baroz et a priori signé un engagement de sa fabrication. Pour le LCIE, j' ai retenu les films Kodak type 2 car il n'avait qu'un film à développer. Des tests ont permis de valider la stabilité des deux émulsions, le faible voile de fond des émulsions combinées et la dynamique de doses en rayonnements gamma.

Un autre point fort pour ce choix a été le fait que les films utilisés par le SCPRI, EDF et aussi par Massiot Philips étaient des films Kodak type 2, et donc bien connus en France. En outre les produits de développement utilisés pour le type 2 étaient les mêmes que ceux du NTA alors que ce n'était pas le cas du type 1. Il fallait juste changer le temps du révélateur entre type 2 et NTA. 
La principale difficulté des films type 2 était de mettre en œuvre une solution pour numéroter les films sans percer la pochette. Différentes méthodes ont été examinées : marquage au rayons $\mathrm{X}$ comme EDF, inscription du numéro par jet d'encre en chambre noire et enfin le collage d'une étiquette sur le film en chambre noire. Il a fallu faire des essais avec différentes natures d'étiquettes. La seule étiquette autocollante ayant bien tenu sur le film lors des développements était composée d'un papier vélin et était autotransposable (colle spéciale). La maquette de l'étiquette attendue a été présentée à un imprimeur capable de les produire avec des séries de découpe, dont deux zones détachables sur la face avant (la seconde étant prévue pour une utilisation d'un film Agfa si le film type 2 venait à disparaître comme le type 1). Cela s'est révélé réalisable par un imprimeur et donc cette solution a été retenue. Elle avait l'avantage de ne plus avoir à marquer les films : l'étiquette imprimée par l'ordinateur était collée partiellement sur le film type 2 inséré dans son porte-écrans. Le transfert de la partie de l'étiquette portant le numéro et faite en chambre noire a nécessité d'y mettre un faible éclairage avec des lampes de sodium en complément des lampes rouge. Il a été fabriqué pour chaque panier des caches munies de petits volets qui se plaçaient sur le panier et que l'opératrice rabattait juste après l'insertion dans une case du film venant d'être coupé et sur lequel était collée la petite zone extraite de l'étiquette.

Une fois cette solution de marquage des films type 2 validée, un cahier des charges a été établi décrivant les besoins pour réussir ce changement en informatisant, cette fois, les analyses des films et les résultats. Pour cela, il a été nécessaire de s'équiper d'un nouvel ordinateur IBM AS-Entry. La Direction du LCIE a validé ce changement mais a lancé aussi un audit par un cabinet externe afin d'optimiser au mieux les ressources humaines et d'organisation de la section. Le matériel a été commandé en partie pour commencer le développement du programme confié toujours à Bernard Goin. L'audit a conduit à organiser l'activité en deux groupes : «Gestion» et «Exploitation», avec une polyvalence du personnel dans chacun des groupes. Pour obtenir cette synergie, les deux groupes devaient avoir leur personnel regroupé chacun dans une grande salle. En conséquence la mutation a nécessité l'organisation d'un déménagement des bureaux, des équipements, de l'ordinateur IBM 34, dans le bâtiment du LCIE nommé REC situé à l'extérieur du LCIE. Des volontaires de la section ont facilité la tâche des déménageurs pour le placement des bureaux et autres. Les salles avaient été préparées avant par les services généraux du LCIE. Ce transfert a été effectué le week-end de façon que le travail de dosimétrie se fasse normalement dès le lundi à la REC; exception faite de travaux de soudage HF et de développement des films. La section «films » est resté six mois à la REC pendant lesquels les anciennes salles ont fait l'objet d'une implantation complète selon le projet convenu avec l'auditeur et la Direction du LCIE. Cela a permis de climatiser l'ensemble par des 
ventilo-convecteurs, y compris le local contenant la cage de Faraday et les soudeuses. Je n'oublie pas que la bonne volonté des opératrices a facilité ce séjour à la REC et aussi l'investissement personnel des deux chefs de Groupe : Chantal Damerval et Annette Noinin.

Durant cette période ont été testés les matériels et les logiciels développés pour cette mutation :

- le nouvel ordinateur IBM AS Entry;

- les logiciels DMT écrits par Bernard Goin intégrant les impressions des étiquettes et le programme d'analyse déterminant la dose via deux tables de correspondance (Rayons X et Cobalt 60) ;

- les densitomètres X-Rite pour la mesure des densités optiques par transmission ayant une sortie RS232), leur interface BIDA « RS232/IBM » pour permettre les acquisitions directes des données du densitomètre dans le logiciel IBM ;

- les lecteurs code-barres, les imprimantes code-barres TEC commandées par sécurité de compatibilité auprès d'un revendeur IBM (figure 10.9);

- la fabrication par les imprimeurs des nouveaux bordereaux d'accompagnement, des rapports de contrôle et des étiquettes autocollantes avec des zones détachables (vélin et colle pour étiquette transposable).

Le retour a été plus simple car le déménagement de retour avait lieu dans des espaces neufs et complètement installés avec un autre mobilier et des nouveaux équipements informatiques. Le transfert a été aussi effectué un week-end de façon que le travail de dosimétrie se fasse normalement dès le lundi dans ce nouvel espace.
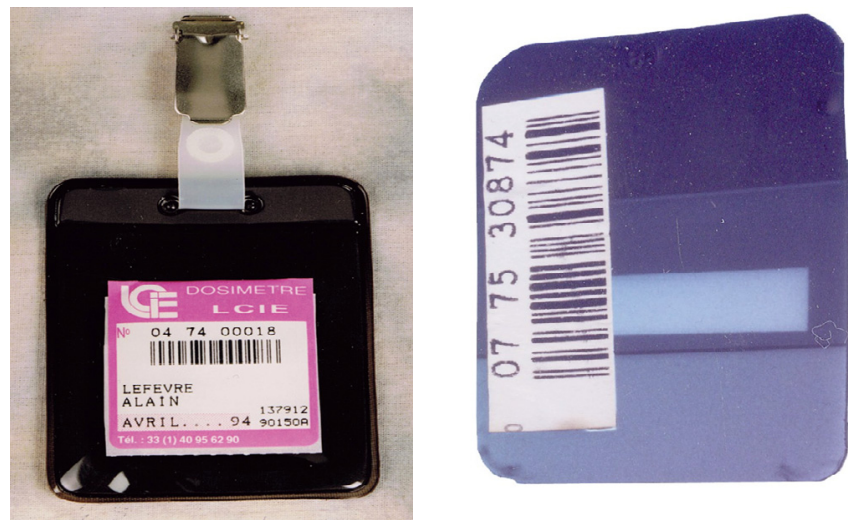

Figure 10.9 - Le Dosimètre film type 7 dans sa pochette et l'étiquette code barres et le film identifié et développé avec son numéro code-barres : dosimètre de poitrine 7, mois juillet, année 1995, $n^{\circ} 30874$. 
La nouvelle organisation a permis de gagner en souplesse. Le service est totalement informatisé en 1994. Le groupe Gestion s'occupe de la gestion des fichiers, des abonnements, de l'envoi des rapports de contrôle, et de l'analyse des comptes (facturation, relances). Une base de données de 180000 noms accessibles en temps réel permet le suivi de chaque personne. Le groupe Gestion assure également la liaison avec l'OPRI et se charge des contacts clients (informations, documentation, courriers clients...). Le groupe Exploitation est divisé en quatre pôles : fabrication/envoi, réception, développement et traitement/analyse. Toutes ces activités sont assurées de manière polyvalente par les membres du groupe. Suite à une application à la lettre de la réglementation par un médecin du travail d'une centrale nucléaire, des nombreux clients ayant des porteurs intervenant en INB avaient résilié leur abonnement malgré la réactivité très appréciée de notre service par ces clients. Une veille de Noël, il était tard et nous assurions un envoi pour un client qui me dit : « je ne comprends pas pourquoi le LCIE ne fait pas une demande d'agrément ». C'était un appel de Jean Claude Zerbib du CEN de Saclay. Je lui ai dit que la demande avait été faite mais était restée sans réponse. Avec son aide j'ai établi un dossier et je l'ai adressé au Ministère du Travail. L'agrément permettant au LCIE d'assurer la dosimétrie externe des intervenants en IBN paraitra au Journal Officiel peu de temps après.

La mise en place des nouvelles grandeurs opérationnelles a commencée au LCIE. Les étalonnages ont été réalisés avec des dosimètres placés sur des fantômes conformément aux recommandations ICRU et ensuite de la norme ISO 4037-3 : 1999(F). J'avais participé aux réunions organisées par le GTN1 portant sur ce sujet. Les résultats du LCIE ont été alors exprimés dans la grandeur « équivalent de dose individuel $\mathrm{Hp}(10)$ » donc l'unité est aussi le millisievert (mSv).

En 1997 le service de Dosimétrie du LCIE a été contacté par EDF qui souhaitait fermer son laboratoire de dosimètre de Saint-Denis. Le contrat à satisfaire, proposé aussi à l'OPRI, consistait à fournir les dosimètres photographiques au personnel $\mathrm{EDF}$, travaillant de manière continue ou intervenant ponctuellement dans les centrales nucléaires. Il a été adressé à EDF une proposition qui respectait leur cahier des charges. Cette offre se calquait sur le fonctionnement de l'EDF en utilisant leur propre numérotation et des bordereaux spécifiques. J'avais trouvé la solution : le numéro EDF serait imprimé sur l'étiquette en lieu et place de la zone destinée au titre « Professeur ou docteur ». Comme d'habitude Bernard Goin m'avait répondu que cela ne posait pas de difficulté et j'avais établi une maquette de dosimètres type 7 destiné à EDF. Après une visite des nouveaux locaux du service de dosimétrie et une présentation de maquettes d'étiquettes et de bordereaux similaires, le LCIE a reçu une réponse favorable à son dossier technique et son offre commerciale et ainsi remporté ce marché auprès d'EDF. 
À nouveau il ne restait qu'à développer un logiciel adapté à leur attente et qui ne concernait que la fourniture de dosimètres de poitrine de type 7 modèle EDF. Pour ce faire, il fallait remplacer l'ordinateur IBM As Entry par un ordinateur plus puissant : un IBM AS 400. J'ai travaillé avec Robert Gadaud, responsable de l'informatique LCIE, qui souhaitait intégrer la gestion informatique de la dosimétrie dans l'ordinateur général du LCIE. Cela a posé une difficulté car les programmes de la dosimétrie étaient écrits depuis 1985 dans des langages IBM toujours en évolution mais restant compatibles entre eux. L'ordinateur du LCIE n'étant pas un IBM, il fallait donc repartir à zéro et cela allait entrainer un coût important pour refaire tous les logiciels pour être intégrés dans le système informatique LCIE. Aussi Robert Gadaud a donné son accord pour la solution IBM AS 400 et pour que le service « films » garde son autonomie informatique. Le programme spécifique à EDF a donc été écrit par Bernard Goin et puis testé en utilisant un jeu de fichiers fournis par EDF et en travaillant aussi avec une personne d'une société de service mandatée par EDF pour les formats des futurs échanges avec Saint-Denis. Aussi afin de satisfaire à une bonne exploitation des 20000 dosimètres EDF, leur gestion a été installée dans une nouvelle salle tous les moyens pour faire leur exploitation : rangement des 60 caisses, expédition, réception avec saisie des codes-barres et mise en chargeur pour le développement.

Pour valider les méthodes et le programme information, un audit très complet du système a été organisé par EDF. Le cycle complet d'une période a fait l'objet d'un test: avec le chargement dans le programme d'un extrait de fichier de commande, l'impression des bordereaux EDF et des étiquettes des dosimètres avec le numéro EDF, le soudage des pochettes dosimétriques pour expédition, le scannage des codes-barres de dosimètres retournés, et leur développement (simulation), la lecture des densités optiques de ces films et la génération des doses, la création du fichier de résultats sur disquette selon le format EDF. Cet audit, piloté entre autres par Alexandre Riedel et Charles Pauron, a fait l'objet d'un rapport complet et noté. Les résultats de l'audit étaient conformes aux attentes d'EDF. La fourniture de dosimètres LCIE a commencé en 1998 ainsi que la mise en place des astreintes EDF pour les jours fériés. Des procédures concernant la gestion des dosimètres EDF ont été établies afin de compléter le système Qualité de la section de dosimétrie. Des intercomparaisons ont été organisées par Alain Biau de l'OPRI pour les dosimètres EDF fournis par le LCIE. D'autre part un arrêté est paru au Journal Officiel citant le LCIE et sa reconnaissance pour cette surveillance. Les fichiers de résultats d'EDF étaient envoyés à Saint-Denis et EDF en effectuait la transmission à l'OPRI.

Depuis 1997 les responsables des services «films» commençaient à comprendre que les émulsions photographiques allaient être remplacées par autre chose. 
Les signes précurseurs étaient les suivants :

- Kodak arrête la fabrication des émulsions nucléaires NTA.

- L'ETCA (devenu SPRA) fait traiter le développement des films Agfa par le CEN/FAR (devenu IRSN) car il n'a plus de chambre noire opérationnelle.

- La fermeture du laboratoire EDF « films » de Saint-Denis.

- Le LCIE a la visite d'un représentant de la société Landauer qui contacte également le CEN/FAR et l'OPRI.

- Les contacts avec le CERN qui utilisaient les émulsions nucléaires NTA pour la détection des neutrons rapides.

J'ai donc commencé à réunir des documentations sur les appareils de lecture de dosimètres TLD ou les détecteurs solides de traces (comme par exemple Autoscan).

Des changements sont intervenus au LCIE à partir de 2001 du fait que le LCIE a été repris par Bureau Véritas. Bertrand Serise a été embauché directement par Bureau Véritas pour prendre en charge l'évolution future de l'activité « films ». La métrologie de rayons X du BNM-LCIE dont j'avais la responsabilité a intégré le LNHB de Saclay. Les générateurs de rayons $X$ de radiodiagnostic et de mammographie ont été installés au LNHB et Marc Denoziere a été embauché par ce laboratoire à l'occasion de ce transfert. Le centre d'étalonnage « Rayonnements ionisants » du LCIE a été repris par BV Véritas.

Pendant la période transitoire de 2001-2002, j'ai assuré la fonction de « Chef de département dosimétrie - rayonnements ionisants au LCIE - Bureau Véritas ». Afin de se centrer sur son métier, Bureau Véritas a cédé l'activité de dosimétrie photographique à Landauer et le laboratoire est devenu LCIE Landauer en 2002.

\subsection{Le service de dosimétrie LCIE Landauer à partir de 2002}

En 2002, le service de dosimètrie a dorénavant comme raison sociale «LCIE Landauer ». C'est une filiale de la société Landaueur dont le siège est à Glenwood (États-Unis). Le président de LCIE Landauer est Bertrand Sérisé. J'ai assuré la fonction de Directeur Technique du LCIE Landauer SAS jusqu'à mon départ en retraite fin mars 2007. La société Philips se recentrant, aussi comme BV, vers son métier principal s'est séparée de son laboratoire de «films »d'Ailly. Elle a été rachetée par Landauer pour être intégrée dans « LCIE Landauer SAS ».

À compter de cette date, la réglementation a commencé à changer pour les laboratoires de dosimétrie. Leur activité a fait l'objet d'un arrêté imposant un agrément par la COFRAC et un avis favorable de l'IRSN. Les actions ont donc été engagées par LCIE Landauer pour obtenir cette accréditation pour les deux sites : celui de Fontenay-aux-Roses et celui d'Ailly. LCIE Landauer a été accrédité par 
le COFRAC (voir l'accréditation COFRAC $\mathrm{n}^{\circ}$ 1-1545 sur le site http://www.cofrac.fr). Le laboratoire a ensuite participé avec succès aux intercomparaisons organisées par l'IRSN. L'autre changement a été la mise en place de SISERI et la transmission des résultats via la plateforme Internet SISERI.

Le fait que la section «films » du LCIE soit devenu «LCIE Landauer» (figure 10.10) a permis d'aller vers un remplacement d'une part, des dosimètres photographiques par des dosimètres OSL InLights et d'autre part, les émulsions nucléaires NTA par des détecteurs solides de traces NEUTRAK.

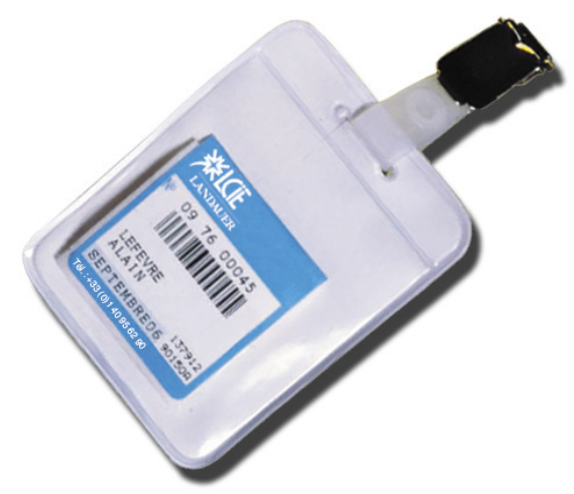

Figure 10.10 - Dosimètre Type 7 avec logo «LCIE Landauer ».

L'écriture des logiciels s'intégrant dans les programmes existants a été à nouveau effectuée par Bernard Goin et a permis d'assurer la dosimétrie des porteurs avec les dosimètres OSL InLight et les détecteurs solides de traces (figure 10.11). Par la suite, la refonte de l'ensemble des logiciels a été confiée à une société informatique et un responsable informatique a été embauché pour gérer l'ensemble des applications. De ce fait Bernard Goin n'est plus intervenu après 2005 et cela a mis fin à cette collaboration de 20 ans qui a permis de faire ces différentes mutations de technologie sans que le service « films » ait de responsable informatique salarié. J'en profite donc pour le remercier à nouveau pour tout ce travail fait en commun, y compris le passage de l'an 2000.

Les activités et les technologies mises à la disposition de ses clients par LCIE Landauer (devenu Landauer EUROPE en 2010) sont présentées dans le site internet ci-après : http://www.landauer-fr.com . Ainsi Landauer EUROPE, premier acteur privé, assure le suivi dosimétrique de plus de 120000 personnes exposées aux 


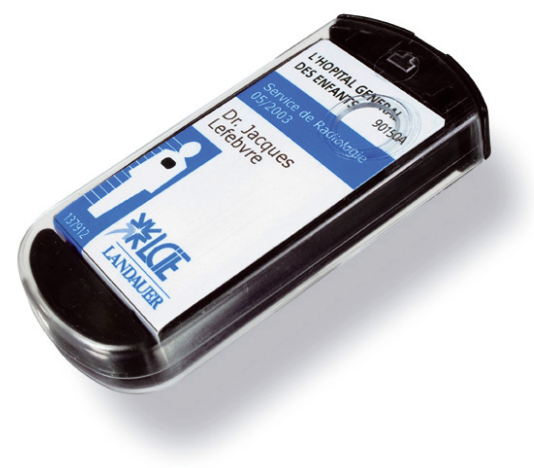

Figure 10.11 - Dosimètre OSL InLight.

rayonnements ionisants. Il faut aussi consulter les autres sites décrivant la technologie OSL : http://www.osldosimetry.com.

Landauer fournit aussi des lecteurs OSL permettant d'équiper des laboratoires de dosimétrie. Ainsi le SPRA a choisi la technologie OSL lorsqu'il a arrêté sa dosimétrie avec des films Agfa. Il s'est équipé de deux lecteurs OSL InLight car les dosimètres peuvent être mis directement dans le lecteur et analysés en quelques secondes. En effet il n'y a pas de préparation avant leur lecture pour les dosimètres OSL, comme c'est le cas avec la technique RPL qui nécessite au préalable un séjour en étuve durant un temps important. De plus le lecteur portable MicroStar est adapté aux situations d'urgence car il permet d'analyser les dosimètres OSL InLight au cours d'exercices militaires ou être d'embarqué dans un sous-marin nucléaire. Par ce choix le SPRA rendait un hommage à Norbert Chassende-Baroz qui avait fait mettre une bande «témoin » sur le film Kodak type 1 afin de pouvoir traiter rapidement les dosimètres urgents sur site. Pour développer le film Kodak type 1, il suffisait de se mettre dans le noir et d'assurer le développement du film dans deux verres (un premier rempli de révélateur et un second de fixateur). Le bon développement se faisait en regardant le noircissement des raies de la bande témoin et l'évaluation d'une irradiation pouvait se faire à l'œil en regardant les trois émulsions fixées sur le support papier.

Ainsi la « boucle est bouclée » en parlant à nouveau des « films » pour finir cette « petite histoire » des films. Pendant plus de 50 ans, en France, ils ont assuré avec succès la surveillance des personnes pouvant être exposées aux rayonnements ionisants. Toutefois il faut garder en mémoire que les « films » sont toujours utilisés dans d'autres pays ; leur faible coût et leur facilité de développement restent leur atout. 


\subsection{L'histoire du « Dosifilm ${ }^{\circledR}$ » de Massiot}

Mon travail avec ce dosimètre a été de courte durée. En effet Philips se recentrant sur son métier a vendu le service de dosimétrie d'Ailly à LCIE Landauer. Ainsi en 2001 je fis ma première visite de ce laboratoire qui était jusqu'à cela un concurrent pour le LCIE sur le marché des « films ». J'ai donc travaillé, dès cette fusion, avec Philippe Pellissier, le responsable de ce laboratoire et aussi avec Dominique Jaegle, qui est venu travailler à Fontenay-aux-Roses. LCIE Landauer ferma son site d'Ailly en 2005, les films étant remplacés par des dosimètres OSL InLight.

\section{Le très connu « Dosifilm »}

Pour moi, Georges Massiot fait partie des grandes figures qui se sont investies dans les nouvelles techniques liées aux rayons X. En effet j'ai vu son nom sur beaucoup d'appareils permettant la mesure de doses :

- des dosimètres intégrateurs Massiot $2 \mathrm{R}$ : destinés aux générateurs de rayons $\mathrm{X}$ de radiothérapie ;

- des microdosimètres Massiot et ses chambres d'ionisation type condensateur. Ce sont des chambres de différents volumes allant de moins $1 \mathrm{~cm}^{3}$ à plus de $600 \mathrm{~cm}^{3}$

- un mesureur de courant Massiot à compensation manuelle et fonctionnant selon le principe de la Balance de Townsend ;

- des écrans fluorescents pour la radioscopie.

En allant sur Internet on trouve de nombreux documents portant le nom de Massiot associé à la radiologie débutante.

Les demandes d'écrans fluorescents pour équiper les installations de radioscopie (installation fixe ou voiturette) ont sans doute conduit à installer une usine pour leur fabrication à Ailly-le-Haut-Clocher, commune du département de la Somme (figure 10.12).

Qui dit image, dit aussi mesure de dose...

Ainsi la société Massiot développa un dosimètre «film » très apprécié dans le milieu médical. La marque déposée « Dosifilm $^{\circledR}$ » était devenue un nom générique employé couramment pour désigner les dosimètres photographiques, tant par les utilisateurs, que par les médecins du travail.

Le film utilisé a changé de type sans doute au cours du temps (film radiologique, film Kodak type 3 puis Kodak type 2). Par contre le filtre dans lequel est inséré le film est resté le même. 

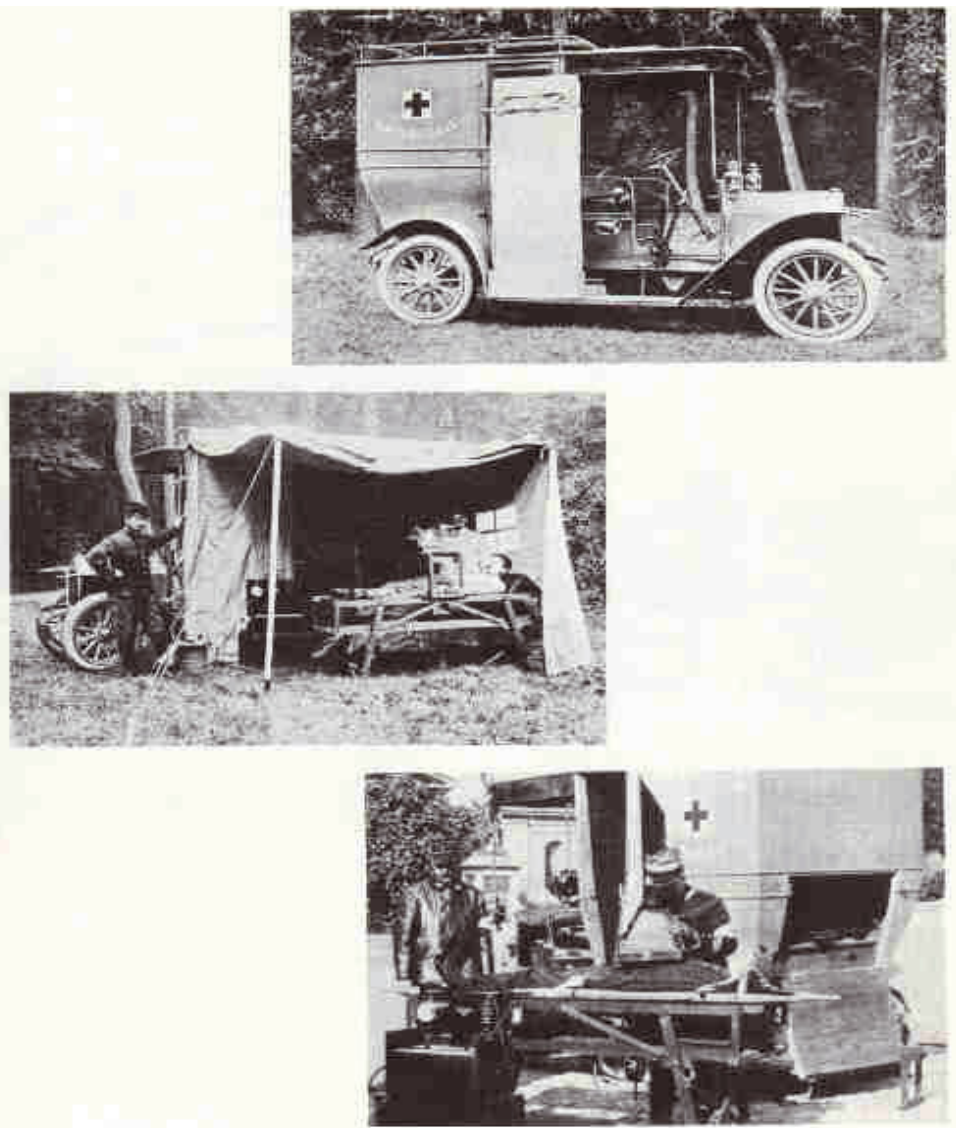

Figure 10.12 - Voiture équipée pour la radiologie Massiot en 1913.

Depuis 1970 le LCIE effectuait des étalonnages de «Dosifilms ${ }^{\circledR}{ }$ pour le laboratoire Massiot d'Ailly.

J'avais eu en main des dosimètres Massiot et je savais que le Dosifilm n'avait que deux plages de lectures : la plage «nue » et la plage sous le « filtre ».

La méthode de détermination des doses à l'aide d'un dosimètre à deux plages avait fait l'objet de différentes publications. Elle était basée sur les travaux du Professeur André Allisy qui a travaillé avec Massiot vers 1955. 
Cette méthode est décrite dans le livre Bases physique de la radiothérapie et de la radiologie par Maurice Tubiana, Jean Dutreix, Andrée Dutreix et P. Jockey (Masson, 1963).

La société Massiot s'est appelée Massiot-Philips puis Philips.

\section{Le site d'Ailly dans la société LCIE Landauer}

En 2001 les activités d'Ailly ont donc été intégrées dans la société LCIE Landauer. Tous les clients fournis avec le «Dosifilm» ont été informés de ce changement. Les documents et les étiquettes des «films» ont été actualisés Landauer (figure 10.13).

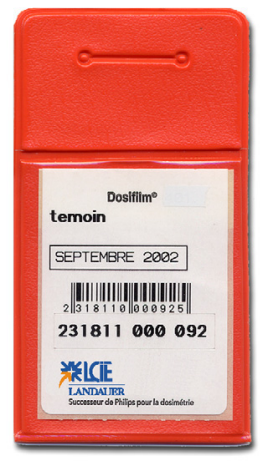

Figure 10.13 - Dosifilm ${ }^{\circledR}$ avec le logo «LCIE Landauer ».

J'ai pris connaissance des modes opératoires du site d'Ailly avec l'aide de son personnel qui était fortement impliqué dans le bon fonctionnement des travaux de développement et de lecture. Philippe Pellissier avait une maîtrise parfaite du logiciel de lecture et tenait compte, avec méthode et rigueur, des variations possibles des sensibilités des films du fait des émulsions Kodak ou de leur développement.

Le laboratoire avait une gestion informatisée utilisant un ordinateur IBM AS 400 relié à des consoles et des imprimantes. Il comportait, entre autres, les équipements techniques suivants : une soudeuse HT pour fermer les pochettes, une chambre noire, un densitomètre à transmission Macbeth interfacé avec l'ordinateur IBM AS 400, un poste de rayons X et un dosimètre PTW et ses chambres d'ionisation. L'installation de rayons $\mathrm{X}$ était destinée à l'irradiation des séries de films étalons qui sont ensuite développés avec les films des porteurs. 
La méthode de détermination des doses était la même depuis 1955 sauf que la dose était maintenant calculée par l'ordinateur. Les mesures de densités optiques étaient faites systématiquement sur les deux plages du film développé par l'opérateur à l'aide du densitomètre Macbeth (figure 10.14).

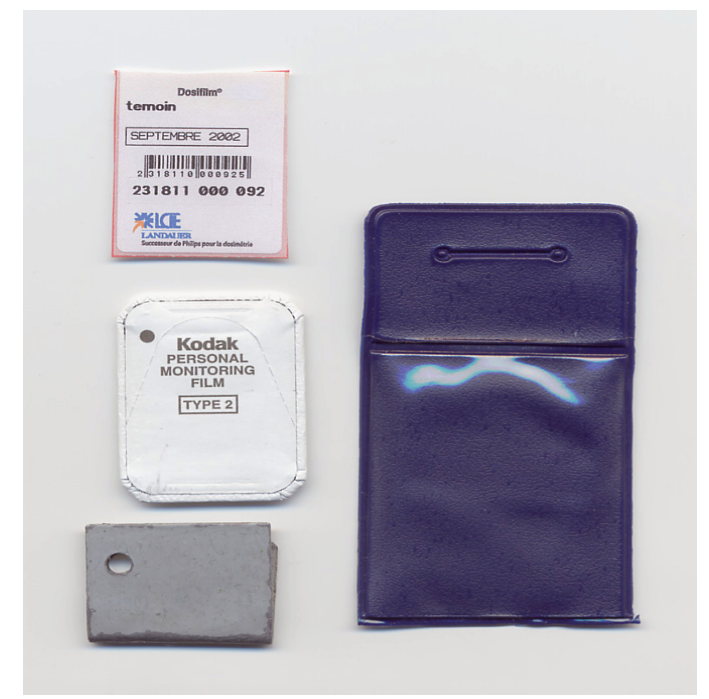

Figure 10.14 - Le film Kodak Type 2 et pochette (voir « filtre » à gauche).

En effet le film type 2 est inséré dans un filtre et cela conduite à avoir deux plages de lecture sur le film développé.

Plage 1 : plage « nue ».

Plage 2: plage sous un filtre ayant une masse surfacique de $1015 \mathrm{mg} / \mathrm{cm}^{2}$ (0,2 $\mathrm{mm}$ de plomb $+0,7 \mathrm{~mm}$ d'étain $+0,2 \mathrm{~mm}$ de plomb $)$.

Le filtre est en fait une petite plaque de ce matériau pliée en deux. Le filtre est le même de part et d'autre du film. En outre le filtre est muni d'un trou circulaire de $3 \mathrm{~mm}$ de diamètre, situé en vis-à-vis sur chaque côté. Ainsi un dosimètre ayant subi une irradiation en position fixe dans un faisceau de rayons $\mathrm{X}$ aura la frontière délimitée par le filtre sur le film développé est nette mais aussi le tour du trou. De plus l'image du trou est nette et ovale, le dosimètre a été exposé en position fixe avec une incidence non de face. 
Jusqu'en 2002, les dosimètres Dosifilm ${ }^{\circledR}$ étaient étalonnés dans l'air pour la grandeur « exposition » dont l'unité est le roentgen. Le passage à l'équivalent de dose $\mathrm{H}$ était alors assuré en appliquant un facteur de qualité $Q=1$. Les étalonnages commandés au LCIE et réalisés dans le centre d'étalonnage COFRAC se faisaient en plaçant les dosimètres dans l'air et en irradiant les dosimètres avec une valeur de la grandeur « exposition » exprimée en C. $\mathrm{kg}^{-1}$. Les valeurs exprimées en C. $\mathrm{kg}^{-1}$ étaient choisies de façon à correspondre à des valeurs entières de l'ancien unité $\left(1 \mathrm{R}=2,58 \cdot 10^{-4} \mathrm{C} \cdot \mathrm{kg}^{-1}\right)$ (voir chapitre 2).

Début 2002, avec Dominique Jaegle, des étalonnages de « Dosifilms » ont été réalisés d'une part dans l'air selon cette méthode et d'autre part, devant fantôme avec comme référence la grandeur opérationnelle « équivalent de dose individuel », $\mathrm{H}_{\mathrm{p}}(10)$. Les coefficients de conversion des faisceaux de rayons $\mathrm{X}$ et de cobalt 60 utilisés étaient ceux donnés dans la norme ISO 4037-3 :1999(F).

Les résultats ont permis de montrer qu'il était possible de conserver la même méthode de calcul pour obtenir la valeur de $\mathrm{H}_{\mathrm{p}}(10)$ à condition bien sûr que les valeurs des densités optiques prises en compte dans la formule proviennent de dosimètres étalonnés en $\mathrm{H}_{\mathrm{p}}(10)$ en étant placés devant un fantôme. La méthode de calcul est donc restée inchangée. Elle n'a pas posé de problème ni pour le traçage des courbes ni pour le calcul des paramètres : pentes et coefficients pour les parties filtrées et non filtrées. C'est aussi à cette période que la réglementation a commencé à changer pour les laboratoires de dosimétrie. Leur activité a fait l'objet d'un arrêté imposant un agrément par la COFRAC et un avis favorable de l'IRSN. Les actions ont donc été engagées par LCIE Landauer pour obtenir cette accréditation pour les deux sites : celui de Fontenay-aux-Roses et celui d'Ailly. LCIE Landauer a été accrédité par le COFRAC pour les deux sites. Ainsi les « Dosifilms » fournis à la clientèle par le site LCIE Landauer ont passés avec succès l'intercomparaison organisée par l'IRSN.

Le site d'Ailly a continué à transmettre ses résultats de doses à l'OPRI via une disquette selon le format défini avec cet organisme. Ce site a été fermé avant la mise en place de SISERI. Les clients d'Ailly ont été informés que des dosimètres OSL InLights remplaceraient les Dosifilms à compter de la période d'avril 2005 pour les abonnements mensuels et aussi pour les trimestriels (période : avril-mai-juin). Le personnel d'Ailly a assuré parfaitement et avec son professionnalisme habituel la réception des Dosifilms des périodes échues, leur développement de périodes, leur lecture et l'envoi des rapports de doses.

Ensuite les moyens techniques nécessaires aux traitements des films retardataires ont été ramenés à Fontenay-aux-Roses : l'ordinateur IBM AS 400, la console interfacée avec le densitomètre Macbeth, le lecteur codes-barres, l'imprimante, des boites de listing pour imprimer les rapports de doses. 
Ayant une connaissance des procédures d'Ailly et aussi du logiciel des doses, tous les Dosifilms retardataires reçus ont été gérés en utilisant le matériel d'Ailly, les films étant développés dans la chambre noire de Fontenay-aux-Roses. Cela s'est fait sans changement car le film du LCIE était le même que celui du Dosifilm : le film Kodak Personal Monitoring cat.8823155 type 2. Les résultats des lectures étaient ensuite imprimés sur les rapports de doses et envoyés aux clients. En fait, les clients d'Ailly retournant régulièrement leur film à la fin de la période, le flot de réception de Dosifilms retardataires, significatif les deux mois suivant les périodes échues, s'est réduit les mois suivants et tari vers octobre 2005. Il a donc été décidé en novembre 2005 d'arrêter leur saisie et de répondre aux clients éventuels que les films d'Ailly n'étaient plus développés.

\section{RÉFÉRENCES}

SFE Bulletin $n^{\circ} 53$ de la Société Française des Électriciens (novembre 1957). Essais du matériel d'électroradiologie au LCIE (Chef de service M. Demanche). Dosimétrie au LCIE (M. Leuzinger).

LCIE (1958a) Bulletin d'information du LCIE n 22 de décembre 1958 (article de M. Leuzinger).

LCIE (1958b) Bulletin d'information du LCIE n 22 de décembre 1958 (article de M. Pierre). 